\title{
Relationships between Length of the Antral Cuff and Postgastrectomy Disorders and Gastric Empting Function in Patients after Pylorus-Preserving Gastrectomy for Early Gastric Cancer
}

\author{
Ryouichi Tomita ${ }^{1,2^{*}}$, Kenichi Sakurai², Shigeru Fujisaki ${ }^{1,2}$ \\ ${ }^{1}$ Department of Surgery, School of Life Dentistry, Nippon Dental University, Tokyo, Japan \\ ${ }^{2}$ First Department of Surgery, School of Medicine, Nihon University, Tokyo, Japan \\ Email: ^rtomita@tky.ndu.ac.jp
}

How to cite this paper: Tomita, R., Sakurai, K. and Fujisaki, S. (2017) Relationships between Length of the Antral Cuff and Postgastrectomy Disorders and Gastric Empting Function in Patients after Pylorus-Preserving Gastrectomy for Early Gastric Cancer. Journal of Cancer Therapy, 8, 867-879.

https://doi.org/10.4236/jct.2017.810076

Received: September 13, 2017

Accepted: October 10, 2017

Published: October 13, 2017

Copyright () 2017 by authors and Scientific Research Publishing Inc. This work is licensed under the Creative Commons Attribution International License (CC BY 4.0).

http://creativecommons.org/licenses/by/4.0/

\begin{abstract}
Objectives: To clarify the optimal length of the antral cuff (LAC) in patients after pylorus-preserving gastrectomy (PPG), we investigated relationships between LAC and postgastrectomy disorder (PGD) such as postprandial abdominal fullness (PAF), and between LAC and gastric empting function (GEF) in PPG patients. Background: The main cause of PGD in PPG patients has been considered to be LAC. Relationships between LAC and PGD and GEF in PPG patients are still unknown. Methods: Of 50 patients who underwent PPG in our hospital from January 2001 to December 2015 were divided into 2 groups [Group A, short LAC of 1.5 to $2.5 \mathrm{~cm}(\mathrm{n}=24)$; Group B, long LAC of 2.6 to $3.5 \mathrm{~cm}(\mathrm{n}=26)$ ]. The relationships among LAC, PGD, and GEF were retrospectively studied. Results: LAC was clearly shorter in group A than group $\mathrm{B}(P<0.01)$. PAF, appetite and food consumption per meal were clearly more favorable in group $\mathrm{B}$ than in group $\mathrm{A}(P<0.01$, respectively). Symptomatic reflux esophagitis (RE), early dumping syndrome, decreased percent body weight for pre-illness, endoscopic RE and endoscopic gastritis in the remnant stomach were more frequent in group A than group B. Gastric stasis in the remnant stomach was clearly more frequent in group $\mathrm{A}$ than group B $(P<0.01)$. GEF with the solid diet in group A was clearly more delayed than in group B $(P<0.01)$. Conclusions: Patients with a short LAC showed worse postoperative QOL and delayed GEF with the solid diet compared with a long LAC.
\end{abstract}




\section{Keywords}

Early Gastric Cancer, Pylorus-Preserving Gastrectomy, Length of the Antral Cuff, Postgastrectomy Disorder, Gastric Empting Function

\section{Introduction}

In clinicopathological analyses, PPG are associated with clearly lower incidences of early dumping syndrome (EDS), gastritis in the remnant stomach and reflux esophagitis (RE) as well as a significant decrease in postoperative malnutrition due to loss of appetite and postoperative body weight loss [1] [2] [3] [4]. Patients after PPG have been reported to have a better postoperative quality of life (QOL) [1] [2] [3] [4]. However, there is a demerit in patients after PPG. The most common demerit in patients after PPG is postprandial abdominal fullness (PAF: early satiety, postprandial fullness and diet limitation are frequent symptoms) due to gastric stasis in the remnant stomach (GSRS) [1] [2] [3] [5] [6] [7]. Frequencies of PAF after PPG have been reported at $30 \%$ to $60 \%$ [1] [2] [3] [5] [6] [7] [8]. PAF has been reported to be closely associated with food intake [9]. Food consumption per meal then becomes insufficient and leads to a marked deterioration of the postoperative QOL, which is one of the major problems in patients after PPG. Therefore, it is considered that PAF may be caused by GSRS due to delay of the gastric emptying function (GEF).

Generally well-known GEF tests are the radioisotope method where gastric emptying of a semisolid diet or solid diet is examined, and the acetaminophen method where gastric emptying of a liquid diet is examined [10] [11]. The radioisotope and acetaminophen methods are physiological and quantitative tests that are noninvasive and cause no bitterness to patients [9]. For the GEF test with a solid diet (rice gruel is substituted in Japanese), a radioisotope method using ${ }^{99 \mathrm{~m}} \mathrm{Tc}$ tin colloid has been used. In order to examine GEF with a liquid diet, the acetaminophen method has been used with orange juice. It is reported that PPG patients show a GEF delay in a solid and semisolid diet [1] [2] [3] [5] [6] [7] [8]. The main cause of postgastrectomy disorders (PGD), especially PAF, due to delayed GEF after PPG has been considered to be the length of the antral cuff (LAC) [2] [11] [12] [13]. However, the optimal LAC still unknown [13].

\section{Aim of the study}

We studied the correlations between LAC and PGD including PAF and GSRS, and between LAC and GEF of the residual stomach in patients who underwent PPG for early gastric cancer.

\section{Patients and Methods}

From January 2001 to December 2015, 50 patients with early gastric cancer without lymph node metastasis in the lower and/or middle stomach visited our 
hospital. Fifty patients ( 30 men and 20 women aged 33 to 79 years with a mean age of 66.7 years) with early gastric M (mucosa) or SM1 (submucosa 1; macroscopically, 1/2 or less submucosal invasion of cancer) cancer of N0 (no lymph node metastasis) underwent PPG with preservation of the pyloric, hepatic and celiac branches of the vagal nerve (PHCV) (M cancer, D1 lymph node dissection; SM1 cancer, D1 $+\alpha$ lymph node dissection in radical curability) [3]. They were divided into 2 groups based on the LAC [Group A $(\mathrm{n}=24)$, LAC was 1.5 to $2.5 \mathrm{~cm}$ [average average $2.0 \pm 0.3 \mathrm{~cm}$ (short LAC)]; Group B $(\mathrm{n}=26)$, LAC was 2.6 to $3.5 \mathrm{~cm}$ [average $3.0 \pm 0.4 \mathrm{~cm}$ (long LAC)] and the correlations between LAC and PGD and between LAC and GEF were compared. Their postoperative courses showed no complications except for one case (wound infection) in group A and 2 cases (wound infection) in group B. No adjuvant therapies including anticancer chemotherapy were applied in either group. The past histories included no diseases of the psychoneurological, metabolic (diabetes mellitus, hypothyroidism) or gastrointestinal systems such as gastrointestinal functional disorders (non-ulcer dyspepsia, irritable bowel syndrome, chronic constipation, and functional intestinal disease) or malignant tumor.

Follow up periods were from 1.5 to 5 years after PPG. This study was performed to each patient at 1.5 years after PPG.

\subsection{Postoperative Interviews and Esophagogastric Scopy}

Symptomes of PAF, appetite, food consumption per meal compared with at normal status, symptoms of RE (e.g., heartburn, feeling of regurgitation, difficulty swallowing), EDS, and percent body weight compared with before illness were inquired of all subjects 1.5 years after operations.

Patients with or without RE, gastritis in the remnant stomach (GRS), and GSRS were also examined by esophagogastric endoscopy.

\subsection{GET for Solid Diet (Radioisotope Method)}

GEF for solid diet (radioisotope method) and GEF for liquid diet (acetaminophen method) were performed in all patients. In GEF for solid diet (radioisotope method), after ${ }^{99 \mathrm{~m}} \mathrm{Tc}$ tin colloid was added to the diet (200 $\mathrm{g}$ of rice gruel with a raw egg) and mixed well, the total quantity was ingested by subjects within 5 minutes in a sitting position. Measurement was performed with a scintillation camera (Hitachi HARP I, Tokyo, Japan). Scanning (imaging) was performed from the abdominal (front) side in a supine position for one minute. The scanning time was from 0 (immediately after the completion of ingestion) to 120 minutes. The data were put into a computer to count radioactivity in the region of interest (ROI) of the stomach. After adjusting for the half-life of ${ }^{99 \mathrm{~m}} \mathrm{Tc}$, the time for the residual rate (\%) of the radioactivity in the stomach on completing ingestion (time 0 ) to reach $50 \%$ of the residual rate after ingestion was calculated, considering the residual rate at time 0 as 100 . Residual rates (\%) at 120 $\mathrm{min}$ in the remnant stomach were also calculated. 


\subsection{GET for Liquid Diet (Acetoaminophen Method)}

After $1 \mathrm{~g}$ of acetaminophen (Yamanouchi, Tokyo, Japan) was dissolved in 250 $\mathrm{ml}$ of orange juice and mixed well, the orange juice was promptly drunk by subjects in a sitting position. Subjects adopted the same supine position as for the radioisotope method for blood collection. This was considered as time 0 , and blood collection was performed at 45 minutes. The blood concentration of acetaminophen was determined with a whole blood concentration determination system (TDX; Abbott, Abbott Park, IL, USA) based on the principle of the immunofluorescence polarization assay.

Subjects discontinued any drugs that might affect gastrointestinal movement from one week before the examination, and did not take any food and drink from the evening (9:00 p.m.) before the examination. The examination was performed from 9:00 a.m. on the following day. GEF with the solid diet was followed by the determination for the liquid diet with an interval of one week.

Informed consent was obtained from all individuals participating in the present study. The present study was approved by the Ethical Committee of Nihon University School of Medicine (Tokyo, Japan).

\subsection{Criteria for Surgery}

Early M or SM1 cancers at the lower and/or middle third of the stomach were selected, where N0 was confirmed by ultrasonic endoscopy, computed tomography and magnetic resonance imaging in preoperative examinations. Furthermore, all cases in this study were unsuited for endoscopic mucosa resection or partial excision of the stomach. Cases in which the remnant stomach would be $1 / 3$ or more were also included (in the present study, the remnant stomach in both groups were 1/3). According to our data, in $M$ cancer the distance from the anal-side margin of the tumor to the pyloric sphincter was $2.5 \mathrm{~cm}$ or more and that in SM1was $3.5 \mathrm{~cm}$ or more, and the resected margin was free from cancer cells, microscopically. In this surgical technique, the pyloric antrum (i.e., length of the pyloric cuff; LPC) 1.5 to $3.5 \mathrm{~cm}$ from the pyloric sphincter was preserved. Regarding lymph node dissection [according to Japanese general rules for Gastric Cancer Study in Surgery and Pathology (the Japanese Classification of Gastric carcinoma), D1 lymph node (numbers 1, 3, 4sb, 4d, 5, 6 and 7) was excised in $M$ cancer while preserving PHCV [3] [14]. SM1 cancer was also excised with $\mathrm{D} 1+\alpha$ (numbers 8a and 9) lymph node while preserving PHCV. D1 lymph nodes except for number 7 are located in the perigastric tissue along the lesser and greater curvatures and in close proximity to the primary tumor. Number 7 lymph nodes are located around the left gastric arteries. Number 8 a lymph nodes in the anterosuperior group are located along the common hepatic artery. Number 9 lymph nodes are located around the celiac artery. In addition, both the infrapyloric artery and vein were preserved.

\subsection{Statistical Analysis}

Statistical analyses were conducted using the non-parametric Kruskal-Wallis test 
with Bonferroni correction (StatView version 5.0 for Macintosh; Abacus Concepts Inc., Berkeley, CA.). Results are expressed as the mean \pm standard deviation (SD). For statistical analysis of the interviews, the chi-squared test was used. For statistical analysis of the percent body weight of the pre-illness weight, the Student's t-test was used. A $P$ value less than 0.05 was regarded as significant.

\section{Results}

\subsection{Characteristics of Patients}

There were no significant differences in patients' characteristics between groups A and B except for LAC. LAC in group A was $2.0 \pm 0.4 \mathrm{~cm}(1.5$ to $2.5 \mathrm{~cm})$ and that in group B $3.0 \pm 0.3 \mathrm{~cm}(2.6$ to $3.5 \mathrm{~cm})$. In addition, LAC was clearly shorter in group $\mathrm{A}$ than group $\mathrm{B}(P<0.01$; Table 1$)$.

Table 1. Characteristics of patients.

\begin{tabular}{|c|c|c|c|}
\hline Factors & Group A $(\mathrm{n}=24)$ & Group B $(n=26)$ & $P$ value \\
\hline Male/Female & $16 / 8$ & $18 / 8$ & ns \\
\hline Age (years) & $66.8(40-79)$ & $64.2(33-78)$ & ns \\
\hline \multicolumn{4}{|c|}{ Depth of cancer invasion } \\
\hline Mucosa & $79.2 \%(19 / 24)$ & $76.9 \%(20 / 26)$ & ns \\
\hline Submucosa & $21.8 \%(5 / 24$ & $23.1 \%(6 / 10)$ & \\
\hline \multicolumn{4}{|c|}{ Lymph node metastasis } \\
\hline No & $100 \%(24 / 24)$ & $100 \%(26 / 26)$ & \\
\hline \multicolumn{4}{|l|}{ Pathological stage } \\
\hline Stage IA & $100 \%(24 / 24)$ & $100 \%(26 / 26)$ & \\
\hline \multicolumn{4}{|l|}{ Lymphnode resection } \\
\hline D1 & $79.2 \%(19 / 24)$ & $80.8 \%(21 / 26)$ & ns \\
\hline $\mathrm{D} 1+\alpha$ & $20.8 \%(5 / 24)$ & $19.2 \%(5 / 26)$ & \\
\hline \multicolumn{4}{|l|}{ PHCV preservation } \\
\hline Preservation & $100 \%(24 / 24)$ & $100 \%(26 / 26)$ & \\
\hline No preservation & $0 \%(0 / 24)$ & $0 \%(0 / 26)$ & \\
\hline \multicolumn{4}{|l|}{ Resected stomach } \\
\hline $1 / 3$ & $100 \%(12 / 12)$ & $100 \%(10 / 10)$ & \\
\hline \multicolumn{4}{|c|}{ Length of antral cuff (cm) } \\
\hline \multicolumn{4}{|c|}{$2.0 \pm 0.43 .0 \pm 0.3<0.01$} \\
\hline \multicolumn{4}{|l|}{ Past history } \\
\hline No & $100 \%(24 / 24)$ & $100 \%(26 / 26)$ & \\
\hline \multicolumn{4}{|c|}{ Postoperative complications } \\
\hline Wound infection & $4.2 \%(1 / 24)$ & $7.7 \%(2 / 26)$ & ns \\
\hline \multicolumn{4}{|c|}{ Postoperative chemotherapy } \\
\hline No & $100 \%(24 / 24$ & $100 \%(26 / 26)$ & \\
\hline
\end{tabular}

Group A: PPG patients with a short length of the antral cuff (LAC); Group B: PPG patents with a long length of LAC; PPG: Pylorus-preserving gastrectomy; PHCV: Pyloric, hepatic and celiac branches of vagal nerve; Age: Data are mean (range); ns: not significant. 


\subsection{Interview}

PAF was observed at a rate of $100 \%(24 / 24)$ in group A and 7.7\% (2/26) in group $B$. In addition, PAF was clearly more frequent in group A compared with that in group B $(P<0.01)$. The remaining cases in group B had no PAF (Table 2$)$. In appetite, "No change compared with at normal status" was observed at a rate of $54.2 \%(13 / 24)$ in group A and $92.3 \%(24 / 26)$ in group B, and was clearly more frequent in group B compared with that in group A $(P<0.01)$. The remaining cases in both groups were "Decreased compared with at normal status" (Table 2). In food consumption per meal compared with at normal status, the level " $2 / 3$ or more compared with at normal status" was observed at a rate of 50.0\% (12/24) in group A and $92.3 \%(24 / 26)$ in group B, being clearly more frequent in group B than that in group A $(P<0.01)$. The remaining cases in both groups were "Less than $2 / 3$ compared with at normal status" (Table 2). Symptoms of RE (e.g., heartburn, feeling of regurgitation, difficult swallowing) occurred in $20.8 \%(5 / 24)$ of group A and 11.5\% (3/26) of group B. They were less frequent in group B compared with group A [not significant (ns)]. The remaining cases in both groups showed no symptomatic RE (Table 2). EDS (systemic symptoms, see ref. 6) occurred in $8.3 \%(2 / 24)$ of group A and 3.8\% (1/26) of group B. In addition, they were less frequent in group B compared with group A (ns). The remaining cases in both groups showed no EDS (Table 2). The percent body weight compared with pre-illness weight was $91.2 \% \pm 5.8 \%$ in group $\mathrm{A}$ and $92.8 \% \pm 4.2 \%$ in

Table 2. Clinical symptoms 1.5 years after PPG.

\begin{tabular}{|c|c|c|c|}
\hline & Group A $(\mathrm{n}=24)$ & Group B $(n=26)$ & $P$ value \\
\hline Postprandial abdominal fullness $100 \%(24 / 24)$ & & $7.7 \%(2 / 26)$ & $<0.01$ \\
\hline \multicolumn{4}{|l|}{ Appetite } \\
\hline No change vs. normal status & $54.2 \%(13 / 24)$ & $92.3 \%(24 / 26)$ & $<0.01$ \\
\hline Decreased vs. normal status & $45.8 \%(11 / 24)$ & $7.7 \%(2 / 26)$ & \\
\hline \multicolumn{4}{|l|}{$\begin{array}{l}\text { Food consumption per meal compared with at } \\
\text { normal status }\end{array}$} \\
\hline $2 / 3$ or more & $50.0 \%(12 / 24)$ & $92.3 \%(24 / 26)$ & $<0.01$ \\
\hline less than $2 / 3$ & $50.0 \%(12 / 24)$ & $7.7 \%(2 / 26)$ & \\
\hline \multicolumn{4}{|l|}{ Symptomatic reflux esophagitis } \\
\hline Negative & $79.2 \%(19 / 24)$ & $88.5 \%(23 / 26)$ & $<0.01$ \\
\hline Positive & $20.8 \%(5 / 24)$ & $11.5 \%(3 / 26)$ & \\
\hline \multicolumn{4}{|l|}{ Early dumping syndrome (systemic symptoms) } \\
\hline Negative & $91.7 \%(22 / 24)$ & $96.2 \%(25 / 26)$ & $<0.01$ \\
\hline Positive & $8.3 \%(2 / 24)$ & $3.8 \%(1 / 26)$ & \\
\hline \multicolumn{4}{|l|}{ Percent body weight for pre-illness weight (\%) } \\
\hline & $91.2 \pm 5.8$ & $92.8 \pm 4.2$ & ns \\
\hline
\end{tabular}

Group A: PPG patients with a short length of the antral cuff (LAC); Group B: PPG patients with a long LAC; PPG: Pylorus-preserving gastrectomy; ns: not significant. 
group B. There was no significant difference between the groups (ns). Body weight decreased in all patients, but the decrease in the percent body weight compared to pre-illness was greater in group A than in group B (Table 2).

$\mathrm{PAF}$, appetite and food consumption per meal were clearly more favorable in group B than group A. Symptomatic RE, EDS and decreased percent body weight compared with pre-illness were more frequent in group A than in group B.

\subsection{Findings of Esophagogastric Endoscopy}

Patients with or without RE, gastritis in the remnant stomach (GRS), and GSRS were examined by esophagogastric endoscopy. Endoscopic RE (Los Angeles classification Grade A) was noted in $16.7 \%$ (2/12) of group A and 3.8\% (1/26) of group B. It was less frequent in group B compared with group A (ns). The remaining cases in both groups had no endoscopic RE (Table 3). GRS occurred at $33.3 \%(8 / 24)$ in group A and $15.4 \%(4 / 26)$ in group B. GRS was more frequent in group A compared with group B (ns). The remaining cases in both groups had no GRS (Table 3). GSRS occurred at $41.7 \%(10 / 24)$ in group A and $23.1 \%(6 / 26)$ in group B. GSRS was clearly more frequent in group A than in group B $(P<$ 0.01). The remaining cases in both groups had no GSRS (Table 3 ).

Endoscopic RE and GRS were more frequently detected in group $A$ than in group B. GSRS was clearly more prevalent in group A than in group B.

\subsection{GET with the Solid Diet (Radioisotope Method)}

The time to $50 \%$ residual rates were $77.4 \pm 8.2$ minutes in group A and $61.8 \pm 6.9$ minutes in group $\mathrm{B}$. The rate in group A was clearly delayed compared with that in group B $(P<0.01$; Table 4).Residual rates (\%) at $120 \mathrm{~min}$ in the remnant stomach were $49.8 \% \pm 8.6 \%$ in group A and $37.6 \% \pm 6.2 \%$ in group B. Residual rates at $120 \mathrm{~min}$ in group A were clearly higher than those in group $\mathrm{B}(P<0.01$; Table 4).

Table 3. Endoscopic findings 1.5 years after PPG.

\begin{tabular}{cccc}
\hline & Group A (n=24) & Group B (n=26) & $P$ value \\
\hline Reflux esophagitis & & & \\
Negative & $83.3 \%(20 / 24)$ & $96.2 \%(25 / 26)$ & $\mathrm{ns}$ \\
Positive & $16.7 \%(4 / 24)$ & $3.8 \%(1 / 26)$ & \\
Gastritis & & & $\mathrm{ns}$ \\
Negative & $66.7 \%(16 / 24)$ & $84.6 \%(22 / 26)$ & \\
Positive & $33.3 \%(8 / 24)$ & $15.4 \%(4 / 26)$ & \\
Gastric stasis in the remnant stomach & & & \\
Negative & $58.3 \%(14 / 24)$ & $76.2 \%(20 / 26)$ & $<0.01$ \\
Positive & $41.7 \%(10 / 24)$ & $23.1 \%(6 / 26)$ &
\end{tabular}

Group A: PPG patients with a short length of the antral cuff (LAC); Group B: PPG patients with a long LAC; PPG: Pylorus-preserving gastrectomy; ns: not significant. 
Table 4. Parameters of gastric emptying functions 1.5 years after PPG.

\begin{tabular}{ccc}
\hline Group A $(\mathrm{n}=24)$ & Group B $(\mathrm{n}=26)$ & $P$ value \\
\hline $77.4 \pm 8.2$ & $61.8 \pm 6.9$ & $<0.01$ \\
& Time to $50 \%$ residual rate in the remnant stomach (minutes) & \\
The residual rates at 120 minutes in the remnant stomach $(\%)$ & \\
$49.8 \pm 8.6$ & $37.6 \pm 6.2$ & $<0.01$ \\
$46.2 \pm 5.7$ & Blood concentration of acetoaminophen at 45 minutes $(\mu \mathrm{g} / \mathrm{ml})$ & \\
$45.5 \pm 4.8$ & $\mathrm{~ns}$ &
\end{tabular}

Group A: PPG patients with a short length of the antral cuff (LAC); Group B: PPG patients with a long LAC; PPG: Pylorus-preserving gastrectomy; ns: not significant.

\subsection{GET with the Liquid Diet (Acetoamonophen Method)}

The blood concentration of acetoaminophen at 45 -minute was $46.2 \pm 5.7 \mu \mathrm{g} / \mathrm{ml}$ in group A and $45.5 \pm 4.8 \mu \mathrm{g} / \mathrm{ml}$ in group B. There were no significant differences in the blood concentration of acetoaminophen at 45-minutes in between groups A and B (Table 4).

According to these results, GEF with the solid food was clearly delayed in group A compared with group B. However, there was no difference in the liquid empting time in between groups.

\section{Discussion}

Even for early gastric cancer located in the middle and/or lower stomach, the distal part of the stomach including the pylorus has conventionally been resected with D2 lymph node dissection without preserving PHCV (i.e., conventional distal gastrectomy) [1]. PGD such as malnutrition due to loss of appetite, weight loss, EDS, RE, or GRS are unfavorable sequelae in patients after conventional distal gastrectomy [1] [2]. To prevent PGD in early gastric cancer, therefore, PPG is now performed wordwide including in Japan. However, some inadequacy of preventing PGD, especially PAF, has been reported in patients one year or more after PPG [1] [2] [3] [4] [6] [7] [11] [12]. The causes of PGD such as PAF in patients after PPG have been studied, focusing on the vagal nerve, infrapyloric vesscels (artery and vein), and LAC [2] [6] [11] [12] [15] [16] [17] [18] [19].

It is considered that PGD, especially PAF with GSRS, after PPG is due to damage to PHCV and infrapyloric vesscels (artery and vein) as a result of skeletonization of the subpyloric region with the subpyloric lymph node dissection [15] [16]. It is also reported that PHCV are necessary for pyloric sphincter functioning in patients after PPG [11] [12]. In recent years the vagal nerves, including hepatic and pyloric branches, have been preserved to prevent PGD including PAF and endoscopic GSRS [13]. Nakabayashi et al. [6] suggested that gastric stasis during the early postoperative period in patients after PPG without preservation of the pyloric branch of the vagal nerve is due to tonic and phasic contractions of the pylorus. In contrast, it was concluded that preservation of the vagal 
nerve such as PHCV is not necessary for gastro-pyloroduodenal motility in patients after PPG [2] [3] [4] [5] [6]. Morita et al. [17] also showed that PAF due to GSRS can be present up to 5 years after PPG even with preservation of the PHCV. Therefore, the roles of PHCV in PPG are not clearly understood. Moreover, it is well-known that the vagal nerves such as PHCV are an important factor in intestinal peristalsis [20]. In addition, it is reported that preservations of the infrapyloric artery and vein are important to prevent the postoperative gastric stasis after PPG [15] [16]. Ischemia around pylorus may lead to pyloric dysfunction. In this study, all subjects were early gastric cancer (M and SM1) without lymph node metastasis. Therefore, we preserved PHCV and both the infrapyloric artery and vein in all PPG patients.

Preserving the pyloric sphincter prevents EDS due to the rapid emptying of food and prevents RE, alkaline GRS due to reflux of duodenal juice into the remnant stomach and esophagus [1]. It was reported that the remnant stomach emptying function was favorable when a $2.0-\mathrm{cm}$ or longer pyloric antrum was preserved, and favorable QOL results were obtained in patients after PPG [2] [11] [12]. Nakane et al. [12] pointed out that in PPG with transection $1.5 \mathrm{~cm}$ proximal to the pyloric ring, PAF and a high incidence of food residue in the remnant stomach after meals were common compared with PPG with transection $2.5 \mathrm{~cm}$ proximal to the pyloric ring, resulting in poor food intake and body weight recovery. They further demonstrated that PPG with transection $2.5 \mathrm{~cm}$ proximal to the pyloric ring was superior to that with transection at $1.5 \mathrm{~cm}$ in terms of postprandial symptoms, food intake, body weight recovery and gastric emptying. They concluded that the length of the retained antrum (i.e., LAC) plays an important role in the motility of the pyloric ring following PPG. According to this data, the size of LAC may be related to the delayed gastric emptying of a diet. Although the LAC gradually increased, from $1.5 \mathrm{~cm}$ during the initial use of the procedure to $3.0 \mathrm{~cm}$ or more currently, its optimal length still remain unclear [13] [17]. As for the interviews in this study, PAF was observed at a rate of $100 \%$ in patients with a short LAC $(1.5$ to $2.5 \mathrm{~cm})$ and at $7.7 \%$ in those with a long LAC (2.6 to $3.5 \mathrm{~cm}$ ). According to this data, the author divided patients after PPG into 2 groups (short LAC and long LAC) in the present studies. To clarify the causes of PGD, especially PAF, studies in PPG patients with preservation of the vagal nerve have been performed focusing on LAC. PAF, appetite and food consumption per meal were clearly better with a long LAC than a short LAC. Symptomatic RE, EDS, and decreased percent body weight versus pre-illness were found more often with a short LAC than with a long LAC.

Antropyloroduodenal coordination is very important to transport gastric contents to the duodenum [21]. GEF, especially PAF, may be a motility disorder such as antropyloroduodenal coordination in patients after PPG. In this study, on findings of esophagogastric endoscopy, endoscopic RE and GRS were more frequenty detected in PPG with a short LAC than with a long LAC. GSRS was clearly more frequent in PPG with a short LAC than in that with a long LAC. In 
accordance with the interviews of PGD and esophagogastric endoscopic findings, PPG patients with a short LAC may experience discoordination of antropyloroduodenal motility compared with those with a long LAC. We suggests that a long LAC may be necessary to coordinate antropyloroduodenal motility for preventing PGD such as PAF.

The postoperative pathophysiology of the GEF of a solid diet in patients after PPG has been assessed in detail. Generally, ${ }^{99 \mathrm{~m}} \mathrm{Tc}$ tin colloid or ${ }^{99 \mathrm{~m}} \mathrm{Tc}-\mathrm{DTPA}$ is used for the solid diet as a nuclide [10]. As the acetaminophen method can be safely performed at a reasonable cost, it is widely used for the determination of the emptying time of a liquid diet instead of the radioisotope method [9]. As a solid diet, we used rice gruel because it is familiar to Japanese people, easy to eat after gastric surgery, and easy to cook and prepare for the experiment. In a previous study, the gastric emptying curve with a rice gruel diet presented a regression line with a high regression coefficient in the control subjects, and author thus considered that there was no problem associated with the use of a rice gruel diet, being the same as a solid diet [22]. The time to $50 \%$ residual rates revealed GEF with a solid diet [9] [10]. In the present study, the author used the time to $50 \%$ residual rates to evaluate GEF in patients after PPG. To examine the liquid diet-emptying function with the acetaminophen method, diets such as milk, liquid food, elemental diet, and orange juice are used. Acetaminophen is not entirely absorbed by the stomach but is promptly absorbed by the small intestine [9]. It is suggested that the blood concentration of acetaminophen at 45 minutes shows the GEF with a liquid meal [9]. We used the same method in this study. Mistiaen et al. [23] also showed that liquid diets are more appropriate for detecting possible rapid gastric emptying rates and EDS. A solid diet test, on the other hand, can be used to study gastric stasis. To clarify the reasons for the persistent PAF and GSRS in the present study, the author investigated the GEF after PPG. The gastric emptying of the solid diet was significantly delayed in PPG with a short LAC compared with a long LAC. However, the gastric emptying of the liquid diet showed no difference between PPG with a short LAC and PPG with a long LAC. Thus, in PPG patients, preservation of a long LAC is considered to be useful to prevent delay of the gastric emptying. It is possible that a feeling of PAF after PPG with a short LAC due to the long-term retention of solid food may occur due to delayed emptying of the solid diet. Therefore, slow emptying of a solid diet in patients after PPG, especially those with a short LAC, may contribute to the sensation of PAF due to GSRS. Regarding GEF with a liquid diet [blood concentration of acetoaminophen at 45 minutes $(\mu \mathrm{g} / \mathrm{ml})$ ], there were also no significant differences among patients after PPG with a short LAC, those with a long LAC, and normal subjects. In this study, GEF with the solid food was clearly delayed in patients with a short LAC compared with that in patients with a long LAC. However, there was no difference in liquid empting time between PPG patients with a short LAC and those with a long LAC.

This was a retrospective study. A randomized control trial comparing the 
postoperative states after PPG with a short LAC and with a long LAC should be performed in the future. Moreover, the mechanism of antropyloroduodenal coordination and/or motility in humans is complicated and its control probably involves the functioning of the neural systems including central, peripheral, and entric nervous systems [7] [21] [24]. Studies from various aspects are thus required to clarify the correlations between these factors and PGD such as PAF with GSRS after PPG in the future.

\section{Conclusion}

PAF, appetite and food consumption per meal were clearly more favorable in PPG patients with a long LAC [3.0 $\pm 0.3 \mathrm{~cm}(2.6$ to $3.5 \mathrm{~cm})]$ than a short LAC [2.0 $\pm 0.4 \mathrm{~cm}(1.5$ to $2.5 \mathrm{~cm})]$. Symptomatic RE, EDS, decreased percent body weight compared with pre-illness, endoscopic RE and GRS were more frequent in PPG patients with a short LAC than in those with a long LAC. GSRS was clearly more prevalent in PPG patients with a short LAC than in those with a long LAC. GEF with solid food was clearly delayed in PPG patients with a short LAC compared with a long LAC. However, there was no difference in the liquid empting time between PPG patients with a short LAC and those with a long LAC. Patients with a short LAC showed a poor postoperative QOL and delayed gastric emptying of a solid food compared to those with a long LAC.

\section{Conflict of Interest}

The authors have read the manuscript and have approved this submission. And there are no no conflicts of interest in association with this study.

\section{Authors Contribution}

Ryouichi Tomita have operated these cases and analyzed all data. Shigeru Fujisaki and Kenichi Sakurai did assistant of the operation.

\section{References}

[1] Park, d.J., Lee, H.J., Jung, H.C., Kim, W.H., Lee, K.U. and Yang, H.K. (2008) Clinical Outcome of Pylorus-Preserving Gastrectomy in Gastric Cancer in Comparison with Conventional Distal Gastrectomy with Billroth Ianastomosis. World Journal of Surgery, 32, 1029-1034. PMID: 18256877

[2] Shibata, C., Sanijo, F., Kakyo, M., Kinouchi, M., Tanaka, N., Sasaki, I., et al. (2012) Currentstatus of Pylorus-Preserving Gastrectomy for Treatment of Gastric Cancer: A Questionnaire Survey and Review of Literatures. World Journal of Surgery, 36, 858-863. PMID: 22350486 https://doi.org/10.1007/s00268-012-1491-6

[3] Song, P., Lu, M., Pu, F., Zhang, D., Wang, B. and Zhao, Q. (2014) Meta-Analysis of Pylorus-Preserving Gatrectomy for Middle-Third Early Gastric Cancer. Journal of Laparoendoscopic \& Advanced Surgical Techniques, 24, 718-728. PMID: 25243417 https://doi.org/10.1089/lap.2014.0123

[4] Namikawa, T., Hiki, N., Kinami, S., Okabe, H., Unshihara, T., Kawahira, H., et al. (2014) Factors That Minimize Postgastrectomy Symptoms Following PylorusPreserving Gastrectomy: Assessment Using a Newly Developed Scale (PGASAS- 
45). Gastric Cancer, 18, 397-406. https://doi.org/10.1007/s10120-014-0366-y

[5] Tomita, R., Takizawa, H. and Tanjoh, K. (1998) Physiological Effects of Cisapride on Gastric Emptying after Pylorus-Preserving Gastrectomy for Early Gastric Cancer. World Journal of Surgery, 22, 35-41. PMID: 24760336 https://doi.org/10.1007/s002689900346

[6] Nakabayashi, T., Mochiki, E., Garcia, M., Haga, N., Suzuki, T., Asao, T., et al. (2002) Pyloric Motility after Pylorus-Preserving Gastrectomy with or without Pyloric Branch of the Vagus Nerve. World Journal of Surgery, 26, 577-583. PMID: 12098048 https://doi.org/10.1007/s00268-001-0270-6

[7] Murakami, H., Matsumoto, H., Kubota, H., Higashida, M., Nakamura, M. and Hirai, T. (2013) Evaluation of Electrical Activity after Vagus Nerve-Preserving Distal Gastrectomy Using Multichannel Electrogastrography. Journal of Smooth Muscle Research, 49, 1-14. PMID: 23832614 https://doi.org/10.1540/jsmr.49.1

[8] Imada, T., Rino, Y., Takahashi, M., Hatori, S., Tanaka, J., Shiozawa, M., et al. (1998) Gastric Emptying after Pylorus-Preserving Gastrectomy in Comparison with Conventional Subtotal Gastrectomy for Early Gastric Carcinoma. Surgery Today, 28, 135-138. PMID: 9525000 https://doi.org/10.1007/s005950050094

[9] Koyama, K., Sasaki, I., Naito, H., Tsuchiya, T., Funayama, Y., Shibata, C., et al. (1995) Gastric Emptying of Semi-Solid Meal in Normal Man with Special Reference to Relationship between Plasma Acetoaminophen Concentrations and Gastric Emptying. Journal of Smooth Muscle Research, 31, 61-65. PMID: 7549050 https://doi.org/10.1540/jsmr.31.61

[10] Christian, P.E., Datz, F.L. and Sorenson, J.A. (1983) Comparison of Tc-99m Labeled Liver and Liver Pate as Markers for Solid-Phase Gastric Emptying. Journal of Nuclear Medicine, 24, 264-268. PMID: 6210765

[11] Nomura, E., Isozaki, H., Fujii, K., Toyoda, M., Niki, M., Sako, S., et al. (2003) Postoperative Evaluation of Function-Preserving Gastrectomy for Early Gastric Cancer. Hepatogastroenterology, 50, 2246-2250.

[12] Nakane, Y., Michiura, T., Inoue, K., Sato, M., Nakai, K. and Yamamichi, K. (2002) Length of the Antral Segment in Pylorus-Preserving Gastrectomy. British Journal of Surgery, 89, 220-224. https://doi.org/10.1046/j.1365-2168.2002.01984.x

[13] Oh, S.Y., Lee, H.J. and Yang, H.K. (2016) Pylorus-Preserving Gastrectomy for Gastric Cancer. Journal of Gastric Cancer, 16, 63-71. https://doi.org/10.5230/jgc.2016.16.2.63

[14] Japanese Gastric Cancer Association (2011) Japanese Classification of Gastric Carcinoma. 3rd English Edition, Gastric Cancer, 14, 101-112. https://doi.org/10.1007/s10120-011-0041-5

[15] Sawai, K., Takahashi, T., Fujioka, T., Minato, H., Taniguchi, H. and Yamaguchi, T. (1995) Pylorus-Preserving Gastrectomy with Radical Lymph Node Dissection Based on Anatomical Variation of the Infrapyloric Artery. The American Journal of Surgery, 170, 1029-1036.

[16] Kiyokawa, T., Hiki, N., Nunobe, S., Honda, M., Ohashi, M. and Sano, T. (2017) Preserving Inflapyloric Vein Reduces Postoperative Gastric Stasis after Laparoscopic Pylorus-Preserving Gastrectomy. Langenbeck's Archives of Surgery, 402, 49-56. https://doi.org/10.1007/s00423-016-1529-6

[17] Morita, S., sassako, M., Saka, M., Fukagawa, T., Sano, T. and Katai, H. (2010) Correlation between the Length of Pyloric Cuff and Postoperative Evaluation after Pylorus-Preserving Gastrectomy. Gastric Cancer, 13, 109-116.

https://doi.org/10.1007/s10120-010-0549-0 
[18] Tokunaga, M., Hiki, N., Fukunaga, T., Ohyama, S., Nunobe, S., Yamada, K., et al. (2011) Is Preservation of Celiac Branch of the Vagal Nerve Effective in Preventing Stasis Following Pylorus-Preserving Gastrectomy? Hepatogastroenterology, 58, 1046-1050.

[19] Morita, S., Katai, H., Saka, M., Fukagawa, T., Sano, T. and Sasako, M. (2008) Outocome of Pylorus-Presrving Gastrectomy for Early Gastric Cancer. British Journal of Surgery, 95, 1429. https://doi.org/10.1002/bjs.6295

[20] Phillips, R.J., Baronowsky, E.A. and Powley, T.L. (1997) Afferent Innervation of Gastrointestinal Tract Smooth Muscle by the Hepatic Branch of the Vagus. Journal of Comparative Neurology, 28, 248-270. https://doi.org/10.1002/(SICI)1096-9861(19970728)384:2<248::AID-CNE6>3.0.CO; 2-1

[21] Stevens, J.E., Doran, S., Russo, A., O’Donovan, D., Feinle-Bisset, C., Rayner, C.K., et al. (2009) Effects of Intravenous Fructose on Gastric Emptying and Antropyloroduodenal Motility in Healthy Subjects. American Journal of Physiology. Gastrointestinal and Liver Physiology, 297, G1274-G1280.

https://doi.org/10.1152/ajpgi.00214.2009

[22] Tomita, R., Fujisaki, S. and Tanjoh, K. (2003) Pathophysiological Studies on the Relationship between Postgastrectomy Syndrome and Gastric Emptying Function at 5 Years after Pylorus-Preserving Distal Gastrectomy for Early Gastric Cancer. World Journal of Surgery, 27, 725-733. https://doi.org/10.1007/s00268-003-6906-y

[23] Mistiaen, W., Van Hee, R., Blockx, P., Bortier, H. and Harrisson, F. (2001) Gastric Emptying Rate for Solid and for Liquid Test Meals in Patients with Dyspeptic Symptoms after Partial Gastrectomy and after Vagotomy Followed by Partial Gastrectomy. Hepatogastroenterology, 48, 299-302.

[24] Spoo, J.W. and Shelton, G.D. (2014) Recurrent Gastric Dilatation and Intestinal Dysmotility Possibly Resulting from Autonomic Neuropathy in a Great Dane. Journal of the American Animal Hospital Association, 50, 221-226.

https://doi.org/10.5326/JAAHA-MS-6176 